Pacific Journal of Mathematics

ANALYTIC CONTINUATION OF INNER 


\title{
ANALYTIC CONTINUATION OF INNER FUNCTION-OPERATORS
}

\author{
Domingo A. Herrero
}

In his "Lectures on Invariant Subspaces", H. Helson has divided the study of the (closed) invariant subspaces of a unilateral shift of countable multiplicity $N$ (regarded as the multiplication by $z$ on $H_{K}^{2}$, the $H^{2}$ Hardy class of analytic functions in the unit disc $D=\{z:|z|<1\}$ with values in a complex separable Hilbert space $K$ of dimension $N$ ) into two main sections: "Full-range subspaces" and "Analytic Range Functions".

An invariant subspace $\mathscr{C}$ is a full-range subspace if it can be written as $\mathscr{C}=U H_{K}^{2}$, where $U$ is an INNER FUNCTION-OPERATOR, i.e, $U(z)$ is a bounded analytic function on $D$ with values in the set of all bounded linear operators in $K$ whose nontangential strong limits $U\left(e^{i x}\right)$ (these limits are well-defined a.e.) are unitary operators in $K$ (a.e). Helson's book contains a study of the analytic properties of an inner function operator in the interior and on the boundary of $D$. In this article the properties of the analytic continuation of these functions outside $D$ are studied; the results also include some information about the cyclic vectors of a $C_{00}$-contraction in a Hilbert space.

Let us state carefully the definitions that we are going to use.

Let $F(z)$ be an analytic function, originally defined on $D$, with values in the complex Banach space $\mathscr{B}$; the "analyticity" and the domain of analyticity (or, the Riemann surface) $R(F)$ of $F$ is defined "via Taylor series" with coefficients in $\mathscr{B}$ converging absolutely in $\mathscr{B}$-norm.

The Riemann surface $R(A)$ of an analytic function with values in the set $\mathscr{L}(\mathscr{B})$ of all bounded linear operators in $\mathscr{B}(A$ will be assumed originally defined on $D$ ) is also defined via Taylor series with coefficients in $\mathscr{L}(\mathscr{B})$, converging absolutely in the norm as operators in $\mathscr{B}$.

As it will be shown in the next section, these conditions can be relaxed.

Let $(\cdot, \cdot)$ and $\|\cdot\|_{K}$ denote the inner product and the norm of $K$, respectively, and let

$$
F(z)=\sum_{n=0}^{\infty} \psi_{n} z^{n}, G(z)=\sum_{n=0}^{\infty} \xi_{n} z^{n}\left(\psi_{n}, \xi_{n} \in K\right)
$$

be two elements of $H_{K}^{2}$; then the non-tangential limits $F\left(e^{i x}\right), G\left(e^{i x}\right)$ 
are well defined for a.e., $e^{i x} \in \partial D$ (the boundary of $D$ ) and the inner product of $F$ and $G$ can be expressed as

$$
<F, G>=\sum_{n=0}^{\infty}\left(\psi_{n}, \xi_{n}\right)=\int_{\partial D}\left(F\left(e^{i x}\right), G\left(e^{i x}\right)\right) d m
$$

$(d m=$ normalized Lebesgue measure on $\partial D)$.

The shift operator $S$ on $H_{K}^{2}$ and its adjoint $S^{\sharp}$ are defined by

$$
\begin{aligned}
& S F(z)=z F(z)=\sum_{n=0}^{\infty} \psi z^{n+1} \\
& S^{\sharp} F(z)=[F(z)-F(0)] / z=\sum_{n=0}^{\infty} \psi_{n+1} z^{n} .
\end{aligned}
$$

We shall denote by $\mathscr{F}^{\sim}(\mathscr{F}$, resp.) the set of all full-range invariant subspaces (inner function-operators, resp.). Let $\mathscr{C}=U H_{K}^{2} \epsilon$ $\mathscr{F}^{\sim}$. $U(\in \mathscr{F})$ is uniquely determined by $\mathscr{l l}$, up to a constant unitary right factor (see [4], Lect. VI). The subspace $\mathscr{K}=\mathscr{C l}^{\perp}$ is clearly invariant under $S^{\sharp}$ and the operator $T: \mathscr{K} \rightarrow \mathscr{K}$ defined by

$$
T F=P(S F) \quad(F \in \mathscr{K})
$$

where $\boldsymbol{P}$ denotes the orthogonal projection of $H_{K}^{2}$ onto $\mathscr{K}$ is a $C_{00^{-}}$ contraction in the sense of Sz.-Nagy and Foiass; moreover, every $\mathrm{C}_{0^{-}}$contraction can be actually represented in this form (see [6], [8]).

The following result is contained in [4], Lect. VII (see also [8], Chap. VI).

THEOREM 1. Let $\mathscr{C}=U H_{K}^{2}(U \in \mathscr{F})$ and let $\mathscr{K}=\mathscr{M}^{\perp}$. Then:

(i) The spectrum $\sigma(T)$ of $T$ (defined by (0.2)) consists of exactly those complex numbers $\lambda \in D$ such that $U(\lambda)$ is not invertible in $K$, and those $\lambda \in \partial D$ such that $U(z)$ cannot be continued analytically to $z=\lambda$.

(ii) If $\lambda \in \partial D$, then $\lambda \notin \sigma(T)$ if and only if all the functions of $\mathscr{K}^{-}$can be continued analytically across $\partial D$ at $z=\lambda$.

(iii) Furthermore, if $R(U)$ is the domain of analyticity of $U(z)$, then $F(z)$ can be continued analytically to $R(U)$, for all $F \in \mathscr{K}$, and

$$
\|F(z)\|_{K} \leqq\left\|\left(I-z T^{*}\right)^{-1}\right\|_{\mathscr{K}}\|F\| \cdot
$$

In the first part of this article we analyze some problems related to the analytic continuation of analytic functions with values in a Banach space.

Using these results, the statements of Theorem 1 are improved in several ways ( $\S \S 2$ and 3 ).

$\S 4$ contains a necessary condition for a function $F \in \mathscr{K}$ to be a cyclic vector for $T^{*}\left(T\right.$ a $C_{00}$ operator), in terms of its analytic con- 
tinuation outside $D$.

Following M. J. Sherman [7] we shall say that the (not necessarily full-range) invariant subspace $\mathscr{l} \subset H_{K}^{2}$ contains the direction of $F \in$ $H_{K}^{2}$ if $f F \in \mathscr{C l}$, for some nonidentically zero scalar function $f$.

If $q H_{K}^{2} \subset \mathscr{L}$, for some inner function $q$, then $\mathscr{C l}$ is called an $I N$-subspace; $\mathscr{C}$ clearly contains all directions and it is actually true that $\mathscr{C l} \in \mathscr{F} \sim$.

Let $(I N) \sim((I N)$, resp.) be the set of all $I N$-subspaces (IN-operators, i.e., those $U \in \mathscr{F}$ such that $U H_{K}^{2}$ is a $I N$-subspace, resp.; see [5], [6]). Then we have the following result:

Proposition 2. ([5], Chap. I; [7]).

(i) Let $\mathscr{l l}=U H_{K}^{2} \in(I N)^{\sim}$; then there exists an inner function $q$ such that: $r H_{K}^{2} \subset \mathbb{l l}\left(r\right.$ an inner function) if and only if $r \in q H^{\infty}$ (i.e., if and only if $q$ divides $r$ ).

(ii) Similarly, if $\mathscr{N}$ is an invariant subspace containing the direction of $F \in H_{K}^{2}$, then there exists an inner function $p$ such that $p F \in \mathscr{N}$ and $f \in H^{\infty}, f F \in \mathscr{N} \Rightarrow f \in p H^{\infty}$.

The function $q$ of (i) is the minimal inner function (mif) of $/ l$ (or $U$ ); the function $p$ of (ii) is the minimal inner function of $F$ with respect to $\mathscr{N}$ (" $p$ is the $\operatorname{mif}(\mathscr{N})$ of $F$ "). In $\S 5$ we shall study the behavior of $p$ in connection with $\mathscr{N}$ and the relation between $q, U$ and $T$; in particular, we shall give a new proof of the following theorem (see [1]; [3]; [5], Chap. V; [8], Chap. VI).

Theorem 3. Let $U \in(I N)$ and let $q$ be its mif. Then:

(i) The domain of analyticity of $U(z)\left(U^{-1}(z)\right.$, resp.) is the same as the domain of analyticity of $q(z)\left(q^{-1}(z)\right.$, resp.); moreover, $U$ and $q$ ( $U^{-1}$ and $q^{-1}$, resp.) have the same kind of isolated singularities.

(ii) In particular, the set $D \cap \sigma(T)$ ( $T$ defined by (0.2)) is precisely the set of all zeroes of $q(z)$ and it agrees with the point spectrum of $T$.

(iii) If $R(q)$ is strictly larger than $D$ and $z \in R(q),|z|>1$, then

$$
\|U(z)\|_{K} \leqq|q(z)| \text {. }
$$

(iv) Moreover, if $I-U^{*}(z) U(z)$ has finite trace for some $z \in D$, then the same result is true for all $z \in R(U)$ and trace norm [I$\left.U^{*}(z) U(z)\right]$ is uniformly bounded on compact subsets of $R(U)$.

Most of the material of this article is contained in the author's thesis ([5], Chap. V).

1. The proofs of Lemma 4, Theorem 5 and Corollary 6 are due to Richard W. Beals. In what follows $\mathscr{B}^{*}$ denotes the (topological) dual 
of the Banach space $\mathscr{B}$.

LEMMA 4. Let

$$
F(z)=\sum_{n=0}^{+\infty} \psi_{n} z^{n}, \psi_{n} \in \mathscr{B}
$$

be an analytic $\mathscr{B}$-valued function defined on $D$ (i.e., the series converges absolutely in $\mathscr{B}$, for all $z \in D$ ) and assume that, for each $\xi \in$ $\mathscr{B}^{*}$, the scalar analytic function $[F(z), \xi]=\xi(F(z))$, can be continued to an analytic function on the open disc $D_{\xi}=\{z:|z|<1+\varepsilon(\xi)\}(\varepsilon(\xi)>0)$.

Then there exists an $\varepsilon>0$ such that the Taylor series (1.1) converges absolutely in $\mathscr{B}$ for all $z,|z|<1+\varepsilon$; i.e., $F(z)$ can be continued to an analytic $\mathscr{B}$-valued function on the open disc of radius $(1+\varepsilon)$.

Proof. By hypothesis, we have

(1) $\lim _{n} \sup \left\|\psi_{n}\right\|_{\mathscr{G}}^{n-1} \leqq 1$,

(2) $\lim _{n} \sup \left|\xi\left(\psi_{n}\right)\right|^{1 / n} \leqq(1+\varepsilon(\xi))^{-1}$, for each $\xi \in \mathscr{B}^{*}$.

Let $\mathscr{B}_{N}^{*}=\left\{\xi \in \mathscr{B}^{*}:\left|\xi\left(\psi_{n}\right)\right| \leqq(1+1 / N)^{-n}, n=0,1,2, \cdots\right\}$.

For each $N, N=1,2, \cdots \mathscr{B}_{N}^{*}$ is a closed subset of $\mathscr{B}^{*}$ and $\mathscr{B}^{*}=$ $\bigcup_{N=1}^{\infty} \mathscr{B}_{N}^{*}$. Moreover, $\mathscr{B}_{N}{ }^{*}$ is clearly convex and equilibrated (i.e., $\lambda \in$ $\left.\partial D, \xi \in \mathscr{B}_{N}^{*} \Rightarrow \lambda \xi \in \mathscr{B}_{N}^{*}\right)$.

According to Baire category theorem, there exists an $N$ such that $\mathscr{B}_{N}^{*}$ has nonempty interior; using this fact and the above observations about $\mathscr{B}_{N}^{*}$, we conclude that there exist an integer $N_{0}$ and $\delta>0$ such that

$$
\left\{\xi:\|\xi\|_{\mathscr{\sigma} *} \leqq \delta\right\} \subset \mathscr{B}_{N_{0}}^{*}
$$

Let $\xi \in \mathscr{B}^{*},\|\xi\|_{\mathscr{}}=1$; it follows that $\left|\xi\left(\psi_{n}\right)\right| \leqq\left(1+1 / \mathrm{N}_{0}\right)^{-n} / \delta$, $n=0,1,2, \cdots$, and therefore

$$
\lim _{n} \sup \left\|\psi_{n}\right\|_{\mathscr{S}^{\prime}}^{1 / n} \leqq\left(1+1 / N_{0}\right)^{-1} .
$$

Thus the statement is true for $\varepsilon=1 / N_{0}>0$.

THEOREM 5. Let $R$ be an open subset of the Riemann sphere $\boldsymbol{C}^{\prime}=\boldsymbol{C} \cup\{\infty\}$ (or, more generally, a Riemann surface) and let $\mathscr{H}_{0}$ be a subset of the space $\mathscr{H}$ of all B-valued functions defined on $R$. Assume that:

and

(1) $\mathscr{H}_{0}$ is convex and closed in the compact-open topology of $\mathscr{H}$,

(2) There exist a countable dense subset $\left\{z_{n}\right\}$ of $\partial R$ and a family $\left\{F_{n} \in \mathscr{C}_{0}\right\}$ such that $z_{n} \notin R\left(F_{n}\right)$, for $n=1,2, \cdots$.

Then: (i) There exists a function $F_{0} \in \mathscr{H}_{0}$ such that $R\left(F_{0}\right)=R$.

(ii) Moreover, there also exists $\xi \in \mathscr{B}^{*}$ such that 


$$
R([F(z), \xi])=R
$$

Proof. For $F, G \in \mathscr{H}$, define

$$
d(F, G)=\sum_{k=1}^{\infty} 2^{-k} \frac{\sup \left\{\|F(z)-G(z)\|_{\mathscr{S}}: z \in M_{k}\right\}}{\left[1+\sup \left\{\|F(z)-G(z)\|_{\mathscr{S}}: z \in M_{k}\right\}\right]},
$$

where $\left\{M_{k}\right\}_{k=1}^{\infty}$ is a sequence of compact subsets of $R$ such that $R=$ $\cup_{k} M_{k}$. As it is well-known, $d$ is a metric on $\mathscr{H}$ and, moreover, $(\mathscr{H}, d)$ is a complete metric space (hence it is a Fréchet space) whose topology coincides with the compact-open topology. Hence $\left(\mathscr{H}_{0}, d\right)$ is a complete metric space.

(i) Let $\left\{z_{n}\right\}$ be as above and set $\mathscr{H}_{n, m, N}=\left\{F \in \mathscr{H}_{0}: F\right.$ extends to an analytic $\mathscr{B}$-valued function on $D_{1 / m}\left(z_{n}\right)=\left\{z:\left|z_{n}-z\right|<1 / m\right\}$, and $\|F(z)\|_{\mathscr{B}} \leqq N$ on $\left.D_{1 / m}\right\}$.

Then $\mathscr{H}_{n, m, N}$ is closed in $\mathscr{H}_{0}$.

Let $F \in \mathscr{H}_{n, m, N}$ and let $F_{n}$ be the function of the statement corresponding to $z_{n}$. Then

$F=\lim .(1-\varepsilon) F+\varepsilon F_{n}=\lim . F_{\varepsilon^{\prime}}$ as $\varepsilon \rightarrow 0$ (in the metric $d$ ) and $F_{\varepsilon} \notin \mathscr{H}_{n, m, N}$ for any $\varepsilon>0$; i.e., $\mathscr{H}_{n, m, N}$ is nowhere dense in $\mathscr{H}_{0}$. It follows from the Baire category theorem that

$$
\mathscr{H}_{0}^{\prime}=\mathscr{H}_{0}-\bigcup_{n, m, N=1}^{\infty} \mathscr{H}_{n, m, N}
$$

is dense in $\mathscr{H}_{0}$. Thus, if $F_{0} \in \mathscr{H}_{0}^{\prime}$, then

$$
R\left(F_{0}\right) \cap \partial R=R\left(F_{0}\right) \cap \text { closure }\left\{z_{n}\right\}=\varnothing .
$$

(ii) Let $F_{0}$ be as above and consider the set

$$
\mathscr{H}_{0}^{\prime \prime}=\left\{\left[F_{0}(z), \xi\right]: \xi \in \mathscr{B}^{*},\|\xi\|_{\mathscr{*} *} \leqq 1\right\} \text {. }
$$

$\mathscr{H}_{0}^{\prime \prime}$ is a convex subset of the space of all scalar analytic functions on $R=R\left(F_{0}\right)$. Assume that $\left\{\xi_{j}\right\}_{j=1}^{\infty}$ is a sequence in the closed unit ball of $\mathscr{B}^{*}$ such that

$$
\lim .\left[F_{0}(z), \xi_{j}\right]=f_{0}(z), \text { as } j \rightarrow \infty,
$$

uniformly on compact subsets of $R$. Passing if necessary to a subsequence, we can assume that $\xi_{j}$ converges in the weak*-topology to some element $\xi_{0} \in \mathscr{B}^{*}$. Clearly we have $\left\|\xi_{0}\right\|_{\mathscr{B}^{*}} \leqq 1$ and $f_{0}(z)=\left[F_{0}(z)\right.$, $\left.\xi_{0}\right]$. Hence $\mathscr{H}_{0}^{\prime \prime}$ is closed and therefore it satisfies the Condition (1) of the statement.

By Lemma 4, we can see that $\mathscr{H}_{0}^{\prime \prime}$ also satisfies the Condition (2). Now the result follows from (i). 
The metric $d$ can be replaced by any metric $\rho$ such that the identity map $\left(\mathscr{H}_{0}, \rho\right) \rightarrow\left(\mathscr{H}_{0}, d\right)$ and the maps $[0,1] \rightarrow \mathscr{H}_{0}$ (defined by $r \rightarrow(1-r) F+r G$, where $F, G$ are two fixed elements of $\left.\mathscr{H}_{0}\right)$ are continuous. A different kind of modification is used in the following

CoRollary $6 .^{1}$ Let $A(z)$ be an analytic function defined on the Riemann surface $R=R(A)$ with values in the set of all bounded linear operators in $\mathscr{B}$. Then there exist $\psi \in \mathscr{B}$ and $\xi \in \mathscr{B}^{*}$ such that $R=$ $R([A(z) \psi, \xi])$.

Moreover, $\psi$ and $\xi$ can be chosen so that, if $\lambda$ is an isolated point of $\partial R$, then $\lambda$ is a pole of order $N$ for $A(z)$ if and only if it is a pole of order $N$ for $[A(z) \psi, \xi]$.

Proof. Let $\mathscr{R}=\mathscr{B} \times \mathscr{B}^{*} ; \mathscr{R}$ is a Banach space with respect to the norm $\|(\psi, \xi)\|_{\mathscr{R}}=\|\psi\|_{\mathscr{A}}+\|\xi\|_{\mathscr{\infty}}$. Observe that $\|A(z)\|_{\mathscr{B}}$ is uniformly bounded on compact subsets of $R$; hence the map $(\psi, \xi) \rightarrow$ $[A(z) \psi, \xi]$ is continuous from $\mathscr{R}$ into the space of all scalar analytic functions on $R$ (compact-open topology).

Let $\mathscr{H}_{0}=\left\{[A(z) \psi, \xi]:\|(\psi, \xi)\|_{\mathscr{R}} \leqq 1\right\}$. We are going to prove that this set of functions satisfies the Condition (2) of Theorem 5; this will follow by the same argument as Lemma 4.

Assume that $A(z)=\sum_{n=0}^{+\infty} A_{n} z^{n}$, where $\left\{A_{n}\right\}_{n=0}^{\infty}$ is a sequence of bounded linear operators in $\mathscr{B}$ such that $\lim _{n}$. sup. $\left\|A_{n}\right\|_{\infty}^{1 / n} \leqq 1$, and that $R([A(z) \psi, \xi])$ includes a disc of radius $1+\varepsilon(\psi, \xi)(\varepsilon(\psi, \xi)>0)$ about $z=0$, for each pair $(\psi, \xi) \in \mathscr{R}$. Applying Lemma 4 to the $\mathscr{B}$-valued functions $[A(z) \psi], \psi \in \mathscr{B}$, it follows that $A(z) \psi$ can be continued to an analytic $\mathscr{B}$-valued function on the disc of radius $1+\varepsilon^{\prime}(\psi)\left(\varepsilon^{\prime}(\psi)>0\right)$.

Let $\mathscr{B}_{N}=\left\{\psi \in \mathscr{B}:\left\|A_{n} \psi\right\|_{\mathscr{B}} \leqq(1+1 / N)^{-n}, n=0,1,2, \cdots\right\}$.

Now the proof that $\lim _{n}$. sup. $\left\|A_{n}\right\|_{s o}^{1 / n} \leqq\left(1+1 / N_{0}\right)^{-1}$ for some integer $N_{0}>0$, follows as in Lemma 4. Therefore, $A(z)$ can be continued analytically to any domain on which all the functions in $\mathscr{H}_{0}$ are analytic. This proves that $\mathscr{H}_{0}$ satisfies the Condition (2) of Theorem 5.

Let $\left\{z_{n}\right\}_{n=1}^{\infty} \subset \partial R,\left\{f_{n}(z)=\left[A(z) \psi_{n}, \xi_{n}\right]\right\} \subset \mathscr{H}_{0}^{\prime}$ and $\mathscr{H}_{n, m, N}$ be defined as in Theorem 5. Define $\mathscr{R}^{1}=\left\{(\psi, \xi):\|(\psi, \xi)\|_{x} \leqq 1\right\}$ and

$$
\mathscr{R}_{n, m, N}=\left\{(\psi, \xi)^{\mathscr{R} \in}:[A(z) \psi, \xi] \in \mathscr{H}_{n, m, N}\right\} .
$$

$\mathscr{R}_{n, m, N}$ is clearly closed in the closed unit ball $\mathscr{R}^{1}$ of $\mathscr{R}$.

Observe that if $f(z)=[A(z) \psi, \xi]$ (where $\left.(\psi, \xi) \in \mathscr{R}^{1}\right)$, then for each $\varepsilon, 0<\varepsilon<1$, the functions $f_{\varepsilon, 1}(z)=f(z), f_{\varepsilon, 2}(z)=\left[A(z)\left((1-\varepsilon) \psi+\varepsilon \psi_{n}\right)\right.$, $\xi], f_{\varepsilon, 3}(z)=\left[A(z) \psi,(1-\varepsilon) \xi+\varepsilon \xi_{n}\right]$ and $f_{\varepsilon 4}(z)=\left[A(z)\left((1-\varepsilon) \psi+\varepsilon \psi_{n}\right)\right.$, $\left.(1-\varepsilon) \xi+\varepsilon \xi_{n}\right]$ belong to $\mathscr{H}_{0}$.

1 The author wants to thank Prof. H. Helson for finding a mistake in the proof of this corollary. 
Since $f(z)=\lim . f_{\varepsilon, j}(z)$, as $\varepsilon \rightarrow 0$ (in the metric $d$; for $j=1,2,3,4$ ) and, for at least one value of $j, f_{\varepsilon, j}$ is not analytic at $z=z_{n}$ for sufficiently small $\varepsilon$, we conclude that $\mathscr{R}_{n, m, N}$ is nowhere dense in $\mathscr{R}^{1}$. It follows as in Theorem 5 that $\mathscr{R}_{0}^{1}=\mathscr{R}^{1}-\bigcup_{n, m, N=1}^{\infty} \mathscr{R}_{n, m, N}$ is dense in $\mathscr{R}^{1}$. Thus we have $R=R(A)=R([A(z) \psi, \xi])$ for all pairs $(\psi, \xi) \in \mathscr{R}_{0}^{1}$.

Finally, if $z_{0}$ is apole of order $N$ for $A(z)$, then the set of pairs $(\psi, \xi) \in \mathscr{R}^{1}$ such that $\left(z-z_{0}\right)^{N-1}[A(z) \psi, \xi]$ is regular at $z=z_{0}$ is closed and nowhere dense in $\mathscr{R}^{1}$. Since $A(z)$ has only countably many singularities, the last statement follows by a refinement of the previous argument.

REMARK. We have actually proved stronger results. In fact we have:

(a) If $F(z)$ is an analytic function with values in $\mathscr{B}$, then

$$
R(F(z))=R([F(z), \xi]),
$$

for all $\xi \in \mathscr{B}^{* \prime \prime}$, where $\mathscr{B}^{* \prime \prime}$ is a $G_{\delta}$-dense subset of $\mathscr{B}^{*}$.

(b) Similarly, if $A(z)$ is an analytic function with values in $\mathscr{L}(\mathscr{B})$, then

$$
R(A(z))=R([A(z) \psi, \xi]),
$$

for all $(\psi, \xi) \in \mathscr{B}^{\prime \prime} \times \mathscr{B}^{* \prime \prime}$, where $\mathscr{B}^{\prime \prime}$ and $\mathscr{B}^{* \prime \prime}$ are $G_{\dot{o}}$-dense subsets of $\mathscr{B}$ and $\mathscr{B}^{*}$, respectively.

2. Let $z \rightarrow z^{*}$ be the "symmetry" of $C^{\prime}$ that fixes $\partial D$; i.e., $0^{*}=$ $\infty ; \infty^{*}=0 ; z^{*}=z \cdot|z|^{-2}$, for $z \neq 0, \infty$.

To every set $R \subset C^{\prime}$, let $R^{*}=\left\{z: z^{*} \in R\right\}$. If $R$ is open and $f(z)$ is analytic on $R$, then $f^{*}(z)=\overline{f\left(z^{*}\right)}$ is analytic on $R^{*}$. The next lemma is a version for operators of the classical reflection principle for scalar functions:

Lemma 7. Let $\Omega \subset C$ be an open disc whose boundary $\partial \Omega$ intersects $\partial D$ orthogonally. Assume that $A(z)$ is an analytic function defined on $D \cap \Omega$ whose values are bounded linear operators in $K$, such that $\|A(z)\|_{K}$ is uniformly bounded there. Then:

(i) $A(z)$ has nontangential strong limits as $z$ approaches $\Gamma=$ $\partial D \cap \Omega$ from the interior of $D \cap \Omega$, for almost every point of $\Gamma$.

(ii) Furthermore, if these limit values are unitary operators in $K$ (a.e., on $\Gamma$ ) and $\left\|A^{-1}(z)\right\|_{K}$ is also uniformly bounded on $D \cap \Omega$, then $A(z)$ can be continued analytically to the domain $\Omega$, by means of the formula

$$
A(z)=\left[A^{*}\left(z^{*}\right)\right]^{-1}, z \in D^{*} \cap \Omega
$$


Proof. (i) The boundary of $D \cap \Omega$ consists of two arcs of circle, so that if $\eta: D \cap \Omega \rightarrow D$ is a conformal mapping, then $\eta$ can be extended to a continuous and one-to-one map from $\bar{\Omega}=$ closure $(D \cap \Omega)$ onto $D \cup \partial D$; this extended $\eta$ is analytic and conformal on $\bar{\Omega}$, except at exactly two points on the boundary (the extreme points of $\Gamma$ ) and it maps sets of positive linear measure on $\partial \bar{\Omega}$ onto sets of positive linear measure on $\partial D$. Moreover, $\eta^{-1}$ has the same properties.

By ([8], Chap. $V-2) A\left(\eta^{-1}(z)\right)$ has nontangential strong limits a.e. Thus (i) follows from the properties of $\eta$.

(ii) Assume that $\|A(z)\|_{K}<M,\left\|A^{-1}(z)\right\|_{K}<M$, for all $z \in D \cap \Omega$. $A^{-1}(z)$ is clearly analytic on $D \cap \Omega$; let $\phi, \psi \in K$; the function $f_{\phi \psi}(z)=$ $\left(A^{-1}(z) \phi, \psi\right)$ is analytic on that domain, and this implies that:

$$
f_{\phi \psi}^{*}(z)=\overline{f_{\phi \psi}\left(z^{*}\right)}=\left(\left[A^{*}\left(z^{*}\right)\right]^{-1} \psi, \phi\right)
$$

is analytic in $z$, for $z \in(D \cap \Omega)^{*}=D^{*} \cap \Omega$. Therefore $\left[A^{*}\left(z^{*}\right)\right]^{-1}$ is analytic in $z$, for $z \in D^{*} \cap \Omega$.

Let $f(z)=f\left(r e^{i x}\right)=f_{\phi \psi}(z), z \in D \cap \Omega$, be defined as above, and let $g\left(r^{-1} e^{i x}\right)=\left(\left[A^{*}\left(r e^{i x}\right)\right]^{-1} \phi, \psi\right)$. If $\Gamma^{\prime}$ is a closed subarc of $\Gamma$, then $r e^{i x} \in \Omega$ for all $e^{i x} \in \Gamma^{\prime}$ and all $r, r_{0}<r<1$. Since $A\left(e^{i x}\right)$ is unitary a.e., we have

$$
\begin{aligned}
& \left\{\int_{\Gamma^{\prime}}\left|f\left(r e^{i x}\right)-g\left(r^{-1} e^{i x}\right)\right|^{2} d m\right\}^{1 / 2} \\
\leqq & \left\{\int_{\Gamma^{\prime}}\left|f\left(r e^{i x}\right)-\left(f e^{i x}\right)\right|^{2} d m\right\}^{1 / 2} \\
+ & \left\{\int_{\Gamma^{\prime}}\left|f\left(e^{i x}\right)-g\left(r^{-1} e^{i x}\right)\right|^{2} d m\right\}^{1 / 2} \rightarrow 0, \text { as } r \rightarrow 1 .
\end{aligned}
$$

By a well known result, and using the fact that $\Gamma^{\prime}$ can be arbitrarily chosen, this implies that the functions $f$ and $g$ continue each other across $\Gamma$ to determine an analytic function on the domain $\Omega$.

Since this result holds for every pair of vectors $\phi, \psi \in K$, it follows from Corollary 6 that $A(z)=\left[A^{*}\left(z^{*}\right)\right]^{-1}$ is the analytic continuation of $A(z)$ across $\Gamma$, to the domain $\Omega$, and it is clear that $\|A(z)\|_{K}<M$ on $\Omega$.

REMARK. It is not hard to see that the boundedness of $A^{-1}(z)$ near $\Gamma$ is also a necessary condition for the analytic continuation of $A(z)$ in the conditions of the lemma. We can say more than that: assume that $f \in H^{\infty}, f(z) \neq 0$ on $D$, and $\left|f\left(r z^{i x}\right)\right|^{-1}=0\left(\exp .\left\{|1-z|^{-t}\right\}\right)$, $t<1$; then $f(z)$ is an outer function ([4], Lect. IV) and therefore, if $U \in \mathscr{F}$, from $\left\|f(z) U^{-1}(z)\right\|_{K} \leqq M$, for $z \in D, z$ in a neighborhood of 1 , we conclude that $\left\|U^{-1}(z)\right\|_{K} \leqq M^{\prime}$, in the same neighborhood. That is,

$$
\left\|U^{-1}(z)\right\|_{K} \neq 0\left(\exp \cdot\left\{|1-z|^{-t}\right\}\right), t<1
$$


in the neighborhood (in $D$ ) of a singularity of $U(z)$ at the boundary of $D$.

The trivial example $U(z)=\exp .\{(z+1) /(z-1)\}$ I shows that the above result is false for $t=1$.

From Theorem 1 and Lemma 7 we get

Theorem 8. Let $U \in \mathscr{F}$; then $R(U)$ is the component containing the origin of the set

$$
C^{\prime} \backslash\left\{z: z^{-1} \in \sigma\left(T^{*}\right)\right\} \text {. }
$$

In particular, $R(U) \subset C^{\prime}$ and $U(z)=\left[U^{*}\left(z^{*}\right)\right]^{-1}$, for all $z \in R(U) \cap$ $D^{*}$.

\section{REMARKS.}

(a) $R(U)$ contains the open circle of radius $\|T\|_{s p}^{-1}\left(\|\cdot\|_{s p}\right.$ denotes the spectral radius).

(b) If

$$
\begin{gathered}
q=b . r \text { is an inner function }, \\
b(z)=\Pi_{k}\left\{\left(\lambda_{k} /\left|\lambda_{k}\right|\right) \quad\left(\lambda_{k}-z\right) /\left(1-\bar{\lambda}_{k} z\right)\right\}
\end{gathered}
$$

is the Blaschke product $\left(\Sigma_{k}\left(1-\left|\lambda_{k}\right|\right)<\infty\right)$ and

$$
r(z)=\exp \cdot\left\{\int_{\partial D}\left(z+e^{i t}\right) /\left(z-e^{i t}\right) d \mu(t)\right\}
$$

$(d \mu$ is a nonnegative Borel measure on $\partial D$, completely singular with respect to $d m$ ) is the singular part, then

(1) If $\partial D \subset \operatorname{supp}(\mu) \cup$ closure $\left\{\lambda_{k}\right\}$, then $R(q)=D$;

(2) If the above inclusion fails, then

$$
R(q)=C^{\prime} \backslash\left[\operatorname{supp}(\mu) \cup \text { closure }\left\{\lambda_{k}^{*}\right\}\right] \text {. }
$$

In the latter case, the points $\lambda_{k}^{*}$ are poles (of the same order as the zero of $q(z)$ at $z=\lambda_{k}$ ) and $q(z)$ is meromorphic in $D^{*}$.

3. Now, we are going to look for a different characterization of $R(U)(U \in \mathscr{F})$, in terms of the elements of $\mathscr{K}$. From the previous results, we get

Corollary 9. Let $\mathscr{A}=U H_{K}^{2} \in \mathscr{F} \sim$. Then there exists $F \in \mathscr{K}=$ $\mathscr{C l}^{\perp}$ such that $R(U(z))=R(z F(z))$. If $\infty$ is an isolated singularity for $U(z)$, then $F$ can be chosen so that $R(U)=R(F)$ if and only if $z U^{-1}(z)$ is singular at $z=0$.

Proof. By Corollary 6, there exists $\phi \in K$ such that $R(U)=$ 
$R(U(z) \phi)$, and $U, U \phi$ have the same kind of isolated singularities. Thus, the same is true (except perhaps for $z=\infty$ ) for: $F(z)=S^{\#} U(z) \phi=$ $[U(z) \phi-U(0) \phi] z^{-1} \in \mathscr{K}$ (see [4], Lect. VIII).

If $\infty \in R(F)$ for all $F \in \mathscr{K}$, then $\infty \in R\left(S^{\sharp} U \dot{\phi}\right)$ for all $\phi \in K$. This implies that $U(z) z^{-1}$ is regular at $z=\infty$; then, by Theorem $8, z U^{-1}(z)$ is regular at $z=0$.

Conversely, if $U^{-1}(z)$ has a pole of order one at $z=0$, then ([5], Chap. III) $\mathscr{K}$ is the direct sum of $\left[B H_{K}^{2}\right]^{\perp}$ and $\left[V H_{K}^{2}\right]^{\perp}$, where $B \in$ $(I N), V \in \mathscr{F}, R\left(B^{-1}(z)\right)=C^{\prime} \backslash\{0\}$ and $0 \in R\left(V^{-1}(z)\right)$.

Moreover, the mif. of $B$ is equal to $z$ and every function in $\left[z H_{K}^{2}\right]^{\perp}$ (which includes $\left[B H_{K}^{2}\right]^{\perp}$ ) is constant. We conclude that $\infty \in R\left(F^{\prime}\right)$ for all $F \in \mathscr{\varkappa}$.

Corollary 10. Let $\mathscr{K}=U H_{K}^{2} \in \mathscr{F}^{\sim}, \mathscr{K}^{\prime}=\mathscr{K}^{\perp}$. Then

$$
\mathscr{K}=\bigvee\{F: R(z F)=R(U)\}
$$

(where V means "the closed subspace spanned by").

Proof. By Corollary 6 and Remarks there exists a $G_{\delta}$ - dense subset $K_{\dot{\delta}}$ of $K$ such that $R(U \dot{\phi})=R(U)$, for all $\phi \in K_{\dot{\delta}}$.

Assume that 0 is not an isolated pole of $U^{-1}(z)$, then we can choose $K_{\delta}$ such that

$$
R(U \phi)=R(U)=R\left[z S^{\sharp(n+1)} U \phi\right]=R\left[z T^{* n}\left(S^{\ddagger} U \phi\right)\right],
$$

for all $\phi \in K_{\delta}$ and all $n=0,1,2 \cdots$, from which the result follows.

If 0 is a pole of (order $N$ of) $U^{-1}(z)$, then there exists $\lambda$ close to 0 such that $\bar{\lambda} \notin \sigma(T)$; then we can choose

$$
K_{\delta}=\left\{\phi \in K: R(U \dot{\phi})=R(U)=R\left(z S^{\sharp} U \dot{\phi}\right)\right\} ;
$$

then

$$
\mathscr{K}=\bigvee_{n=0}^{\infty}\left\{\left(T^{*}-\lambda\right)^{n}\left(S^{\sharp} U \dot{\phi}\right): \phi \in K_{\delta}\right\}
$$

and

$$
R\left[z\left(T^{*}-\lambda\right)^{n}\left(S^{\ddagger} U \phi\right)\right]=R\left[z S^{\sharp} U \phi\right]=R(U) .
$$

4. We do not know whether a cyclic vector $F$ of $T^{*}$ (whenever it exists!) satisfies the condition $R(z F)=R(U)$ or not. However, this result is true if, e.g., $\sigma(T)$ is totally disconnected. In fact, we have:

Proposition 11. Let $\mathscr{K}=U H_{K}^{2} \in \mathscr{F} \sim, \mathscr{K}=\mathscr{K}^{\perp}$ and assume that $\sigma(T)=A_{1} \cup A_{2}$, where $A_{1}$ and $A_{2}$ are two disjoint nonempty compact subsets of $\bar{D}$ : Let $F \in \mathscr{K}$ be a function such that $R\left(z F^{\prime}\right) \supset A_{2}^{*}$ and let $\mathscr{K}_{F}^{*}=\bigvee_{n=0}^{\infty}\left(T^{* n} F\right)$. 
Then $\mathscr{K}_{F}^{*} \neq \mathscr{K}$; i.e., $F$ cannot be a cyclic vector for $T^{*}$.

Proof. By hypothesis, there exists a rectifiable union of closed arcs $\Gamma \subset C^{\prime}-\left(A_{1}^{*} \cup A_{2}^{*}\right)$ containing $A_{2}^{*}$ in its interior and separating $A_{1}^{*}$ from $A_{2}^{*}$. Since $\Gamma$ is a compact set, it follows from Theorem 1 , (iii) that there exists a constant $M$ such that

$$
\|G(z)\|_{K} \leqq M\|G\| \text {, for all } G \in \mathscr{K}^{r} \text { and all } z \in \Gamma \text {. }
$$

Let $G \in \mathscr{Y}_{F}^{*}$; then there is a sequence of polynomials $P_{\nu}(z)=$ $\sum_{k=0}^{N_{\nu}} c_{k}^{\nu}\left(T^{* k} F^{\prime}\right) \in \mathscr{K}_{F}^{*}$ converging to $G$ in $\mathscr{K}^{2}$-norm, as $\nu \rightarrow \infty$. This fact, and (4.1) imply that

$$
\left\|z\left[P_{\nu}(z)-G(z)\right]\right\|_{K} \rightarrow 0 \text {, as } \nu \rightarrow \infty \text {, uniformly on } \Gamma \text {. }
$$

It is clear that $R\left(z P_{\nu}\right) \supset R(z F)$ for all $\nu$. Thus, by (4.2) and the modulus maximum principle,

$$
\left\|z P_{\nu}(z)-z G(z)\right\|_{K} \rightarrow 0 \text {, as } \nu \rightarrow \infty \text {, uniformly on } A_{2}^{*} \text {. }
$$

Therefore $A_{2}^{*} \subset R(z G)$.

On the other hand, (by Corollary 9) there exists $F_{0} \in \mathscr{K}$ such that $R\left(z F_{0}\right)=R(U) \not \supset A_{2}^{*}$. Hence $F_{0} \in \mathscr{K}^{\sim}-\mathscr{K}_{F}^{*}$.

5. Finally, we are going to analyze the domain of analyticity of the $\operatorname{mif}(\mathscr{C}) q$ of a constant function.

THEOREM 12. Let $\mathscr{M}=U H_{K}^{2} \in \mathscr{F} \sim$ and assume that $\mathscr{M}$ contains the direction of $\phi \in K$, with mif $(\mathscr{C}) q$. Let $F \in \mathscr{K}^{\prime}=\mathscr{M}^{\perp}$; then:

$$
R(U) \subset R(q) \subset R[z(F, \phi)] \text {. }
$$

The proof is contained in the following two lemmas:

Lemma 13. Let $\mathscr{C}=U H_{K}^{2} \in \mathscr{F} \sim$ and assume that $q \phi \in \mathscr{M}$, where $q$ is the mif $(U)$ of $\phi \in K^{1}$. Then $R(q) \supset R(U)$.

Proof. Let $F \in H_{K}^{2}$ be the function such that $q \phi=U F$. The minimality of $q$ implies that there is no nonconstant inner function $p$ such that $\bar{p} F \in H_{K}^{2}$.

Let $p$ be any inner function such that $p(z) \neq 0$ for all $z \in D-$ $[R(U)]^{*}$ and $p(z)$ is analytic in (a neighborhood of) $\partial D-R(U)$. It is clear from $F(z)=q(z) . U^{-1}(z) \phi\left(z \in R(U) \cap[R(U)]^{*}\right)$ that $p$ divides $q$ if and only if $\bar{p} F \in H_{K}^{2}$. Hence $p$ must be constant and therefore $q(z)$ and $F(z)$ are analytic on $R(U) \cap[R(U)]^{*}$ : it follows that they are analytic on $R(U)$. Thus $R(q) \supset R(U)$. 
LEMma 14. Let $\mathscr{C l}$ be an invariant subspace and let $F \in \mathscr{K}=$ $\mathscr{C}^{\perp}$. Assume that $q \phi \in \mathscr{L}$, where $q$ is the mif $(\mathscr{C})$ of $\phi \in K^{1}$. Then $R[z(F, \phi)] \supset R(q)$.

Proof. Let $F, \phi, q$ be as above and assume that $R(q) \neq D$; we have:

(1) $f=z(F, \phi) \in H_{0}^{2}$, and

(2) $q \bar{f}=q(\phi, F)=(q \phi, F) \in H_{0}^{2}$, because $q \phi \in \mathscr{C}$, where $H_{0}^{2}$ denotes the subspace of $H^{2}$ consisting of all those functions having mean value zero.

Therefore $h\left(e^{i x}\right)=\bar{q}\left(e^{i x}\right) f\left(e^{i x}\right)$ can be continued to an analytic function on $D^{*}(h(\infty)=0)$. Thus, $g=z q h$ is analytic on $R(q) \cap D^{*}$ and (as in Lemma 7) $f$ and $g$ continue each other across any arc of $\partial D \cap R(q)$. Hence $R(q) \subset R[z(F, \phi)]$.

Now, Theorem 3 is just a corollary of the above results:

In fact, if $U \in(I N)$ with mif. $q$, then the set

$\{\phi \in K: \operatorname{mif}(U)$ of $\phi$ is equal to $q\}$

is a $G_{\delta}$-dense subset of $K$ (see [5], Chap. V; [6]). Using this fact and Corollary 6 and Remarks it is not hard to prove the existence of a vector $\phi \in K$ and a function $F \in \mathscr{K}$ such that:

(1) the mif $(U)$ of $\phi$ is equal to $q$;

(2) $\quad R(U)=R[z(F, \phi)]$.

Hence, by Theorem 12,

$$
R(U) \subset R(q) \subset R[z(F, \phi)]=R(U) .
$$

It follows from the results of [5], Chap. III (see also [2]), that $U^{-1}(z)$ and $q^{-1}(z)$ have the the same kind of poles inside $D$, and every isolated singularity of these (operator-valued and scalar-valued) two functions lying on $\partial D$ is necessarily an essential singularity, as it trivially follows from Lemma 7 .

From these observations and Theorem 8 we get (i).

The statement (ii) follows from the results of [5], Chap. III (see also [8], Chap. VI).

Finally, since $q U^{-1} \in(I N)$, it follows that if $R(q)$ is strictly larger than $D$ and $\lambda^{*} \in D^{*} \cap R(q)$, then (by Theorem 8 ) we get (0.3):

$$
\left\|U\left(\lambda^{*}\right)\right\|_{K}=\left\|U^{-1}(\lambda)\right\|_{K} \leqq\left|q(\lambda)^{-1}\right|=\left|q\left(\lambda^{*}\right)\right| \text {. }
$$

This result can be also obtained from the canonical formulas for $I N$-operators (see [2]; [3]; [5], Chap. IV). Moreover, the analytic continuation of $U(z)$ outside $D$ (whenever this is possible) is provided by the same canonical formulas. 
If $I-U^{*}(z) U(z)$ has finite trace for some $z \in D$, then we can use the formulas of [1] in order to see that, in this case, trace norm [I$\left.U^{*}(z) U(z)\right]$ is bounded (uniformly on compact subsets!) for all $z \in R(U)$. The proof of Theorem 3 is complete now.

REMARKs. (a) If $q$ is a singular inner function and $c>0$ is the total mass of the singular measure of $r(z)$ (as in (3.1), (3.3)), then

$$
|q(z)|=0\left(\exp \cdot\left\{c(|z|-1)^{-1}\right\}\right)
$$

(as $z \in D^{*}$ approaches $\partial D$ ) and $\|U(z)\|_{K}$ has the same property.

(b) If $\operatorname{dim} . K=N<\infty$, then the behavior of $U$ and $q$ is even closer. In fact, in that case we have

$$
\mid \text { det. }\left.U(z)\right|^{-1} \leqq\left\|U^{-1}(z)\right\|_{K}^{N} \leqq\left|q(z)^{-N}\right| \leqq|\operatorname{det} . U(z)|^{-N},
$$

for all $z \in D$ such that $q(z) \neq 0$, and the reversed inequalities hold for all $z \in D^{*} \cap R(q)$.

(c) The similarities between the $I N$-operator $U$ and its mif. $q$ break down at some points if dim. $K$ is not finite.

Example. Define $U \in(I N)$ by $U \phi_{n}=b\left(e^{i x}, \lambda_{n}\right) \phi_{n}, n=1,2, \cdots$ where $\left\{\lambda_{n}: 0<\lambda_{n}<1\right\}_{n=1}^{\infty}$ is an increasing Blaschke sequence; then the mif. of $U$ is $q(z)=I I_{n} b\left(z, \lambda_{n}\right)$.

If $\lambda_{n}$ tends to 1 slowly enough, then we can get $\lim . q(z)=0$, as $z \rightarrow 1$ along any of the circles passing through the points $\{-1, i r,+1\}$, $0<r<1$. However, by a straightforward computation, we can check that $\left\|U^{-1}(z)\right\|_{K} \leqq r^{-1}$ on the circle passing through $i r$.

Added in Proof. After this paper was written, the author has received the preprint "Boundedness from measure theory", by Henry Helson. In this article, Prof. H. Helson gives an essentially different proof of Lemma 4 and its corollaries, among other results.

\section{REFERENCES}

1. I. P. Ginsburg, On multiplicative representation of bounded analytic operatorfunctions, Dokl. Akad. Nauk., SSSR, 170. No. 1 (1966), 23-26 (in Russian).

2. - Multiplicative representation of operatorfunctions of bounded type, Uspehy Mat. Nauk., XXII, No. 1 (1967), 165-167 (in Russian).

3 . - Multiplicative representation and minorants of bounded analytic operatorfunctions, Functional Analysis and its Appl., 1 (1967) (translated from Russian by "Consultants Bureau" New York).

4. H. Helson, Lectures on Invariant Subspaces, Academic Press, New York, 1964.

5. D. A. Herrero, Inner function-operators, Thesis, Univ. of Chicago, 1970.

6. - The exceptional subset of a $C_{0}$-contraction (to appear in Trans. Amer. Math.

Soc.). 
7. M. J. Sherman, Invariant subspaces containing all analytic directions, J of Functional Analysis, 3 (1969), 164-172.

8. B. Sz.-Nagy, and C. Foias, Analyse harmonique des opérateurs dans l'espace de Hilbert, Masson et Cie., Akadémiai, Kiadó, Budapest, 1967.

Received November 10, 1970 and in revised form June 21, 1971. This work was supported by NSF Grants GP-14255 and GU-3171.

\section{UNIVERSITY OF CHICAGo}

AND

State University of New York at Albany 


\title{
PACIFIC JOURNAL OF MATHEMATICS
}

\section{EDITORS}

\author{
H. SAMELSON \\ Stanford University \\ Stanford, California 94305 \\ C. R. HobBY \\ University of Washington \\ Seattle, Washington 98105
}

J. DugundJI

Department of Mathematics

University of Southern California

Los Angeles, California 90007

RICHARD ARENS

University of California

Los Angeles, California 90024

\section{ASSOCIATE EDITORS}
E. F. BECKENBACH
B. H. NeumanN
F. WOLF
K. YOSHIDA

\section{SUPPORTING INSTITUTIONS}

\author{
UNIVERSITY OF BRITISH COLUMBIA \\ CALIFORNIA INSTITUTE OF TECHNOLOGY \\ UNIVERSITY OF CALIFORNIA \\ MONTANA STATE UNIVERSITY \\ UNIVERSITY OF NEVADA \\ NEW MEXICO STATE UNIVERSITY \\ OREGON STATE UNIVERSITY \\ UNIVERSITY OF OREGON \\ OSAKA UNIVERSITY
}

\author{
UNIVERSITY OF SOUTHERN CALIFORNIA \\ STANFORD UNIVERSITY \\ UNIVERSITY OF TOKYO \\ UNIVERSITY OF UTAH \\ WASHINGTON STATE UNIVERSITY \\ UNIVERSITY OF WASHINGTON
AMERICAN MATHEMATICAL SOCIETY
NAVAL WEAPONS CENTER

The Supporting Institutions listed above contribute to the cost of publication of this Journal, but they are not owners or publishers and have no responsibility for its content or policies.

Mathematical papers intended for publication in the Pacific Journal of Mathematics should be in typed form or offset-reproduced, (not dittoed), double spaced with large margins. Underline Greek letters in red, German in green, and script in blue. The first paragraph or two must be capable of being used separately as a synopsis of the entire paper. The editorial "we" must not be used in the synopsis, and items of the bibliography should not be cited there unless absolutely necessary, in which case they must be identified by author and Journal, rather than by item number. Manuscripts, in duplicate if possible, may be sent to any one of the four editors. Please classify according to the scheme of Math. Rev. Index to Vol. 39. All other communications to the editors should be addressed to the managing editor, Richard Arens, University of California, Los Angeles, California, 90024.

50 reprints are provided free for each article; additional copies may be obtained at cost in multiples of 50 .

The Pacific Journal of Mathematics is published monthly. Effective with Volume 16 the price per volume (3 numbers) is $\$ 8.00$; single issues, $\$ 3.00$. Special price for current issues to individual faculty members of supporting institutions and to individual members of the American Mathematical Society: $\$ 4.00$ per volume; single issues $\$ 1.50$. Back numbers are available.

Subscriptions, orders for back numbers, and changes of address should be sent to Pacific Journal of Mathematics, 103 Highland Boulevard, Berkeley, California, 94708.

\section{PUBLISHED BY PACIFIC JOURNAL OF MATHEMATICS, A NON-PROFIT CORPORATION}

Printed at Kokusai Bunken Insatsusha (International Academic Printing Co., Ltd.), 270, 3chome Totsuka-cho, Shinjuku-ku, Tokyo 160, Japan. 


\section{Pacific Journal of Mathematics}

\section{Vol. 40, No. $2 \quad$ October, 1972}

Louis I. Alpert and L. V. Toralballa, An elementary definition of surface area in $E^{n+1}$ for smooth surfaces...........................

Eamon Boyd Barrett, A three point condition for surfaces of constant mean curvature........................................

Jan-Erik Björk, On the spectral radius formula in Banach algebras ....... 279

Peter Botta, Matrix inequalities and kernels of linear transformations . . . . 285

Bennett Eisenberg, Baxter's theorem and Varberg's conjecture ........... 291

Heinrich W. Guggenheimer, Approximation of curves .............. 301

A. Hedayat, An algebraic property of the totally symmetric loops associated with Kirkman-Steiner triple systems ....................... 305

Richard Howard Herman and Michael Charles Reed, Covariant representations of infinite tensor product algebras ................

Domingo Antonio Herrero, Analytic continuation of inner

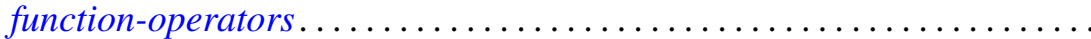

Franklin Lowenthal, Uniform finite generation of the affine group......... 341

Stephen H. McCleary, 0-primitive ordered permutation groups .......... 349

Malcolm Jay Sherman, Disjoint maximal invariant subspaces .......... 373

Mitsuru Nakai, Radon-Nikodým densities and Jacobians .............. 375

Mitsuru Nakai, Royden algebras and quasi-isometries of Riemannian manifolds. . .

Russell Daniel Rupp, Jr., A new type of variational theory sufficiency

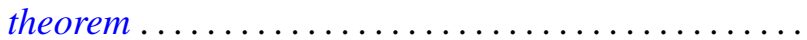

Helga Schirmer, Fixed point and coincidence sets of biconnected multifunctions on trees..........................

Murray Silver, On extremal figures admissible relative to rectangular

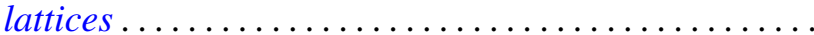

James DeWitt Stein, The open mapping theorem for spaces with unique segments ...

Arne Stray, Approximation and interpolation

Donald Curtis Taylor, A general Phillips theorem for $C^{*}$-algebras and some applications

Florian Vasilescu, On the operator $M(Y)=T Y S^{-1}$ in locally convex algebras...

Philip William Walker, Asymptotics for a class of weighted eigenvalue problems...

Kenneth S. Williams, Exponential sums over $\mathrm{GF}\left(2^{n}\right)$. 